\title{
SCIRR39 Promotes Neurite Extension via RhoA in NGF-Induced PC12 Cells
}

\author{
C.F. Zhao Y.Liu Y.L. Ni J.W. Yang H.D. Hui Z.B.Sun S.J. Liu
}

State Key Laboratory of Proteomics, Department of Neurobiology, Institute of Basic Medical Sciences, The Academy of Military Medical Sciences, Beijing, PR China

\section{Key Words}

Spinal cord injury, regeneration $\cdot$ Fyn $\cdot$ RNH1

\begin{abstract}
SCIRR39 is an identified upregulated gene in rat primary neuron injury and/or regeneration process with roles largely unexplored. Using real-time quantitative PCR, Western blotting and immunofluorescence, SCIRR39 expression was detected in normal PC12 cells and upregulated in differentiated cells. The results of cell proliferation by Cell Counting Kit and cell cycle by flow cytometry indicated that SCIRR39 inhibited cell proliferation and induced the decrease in S phase. Importantly, immunofluorescent and RhoA pull-down assays showed that SCIRR39 strongly affected the neurite extension of NGFtreated PC12 cells through a RhoA-dependent mechanism, but the truncated mutants of SCIRR39 containing a truncation from 141AA to 211AA or from 397AA to 424AA failed to mock the SCIRR39 effect on neurite extension. Moreover, change of SCIRR39 expression in NGF-treated PC12 cells regulated the expression and phosphorylation of Fyn, a regulator of RhoA activity, but not the expression of ROCK II protein. Finally, immunofluorescence and RhoA pull-down assays revealed that obvious inhibition of neurite extension by SCIRR39 shRNA was reversed by RhoA inhibitor C3-transferase. Our results indicated that SCIRR39 increased the neurite extension in NGF-treated PC12 cells via RhoA, suggesting that SCIRR39 contributes to the regeneration of neuron injury by specifically altering the differentiation program.
\end{abstract}

C 2013 S. Karger AG, Basel

\section{KARGER}

(c) 2013 S. Karger AG, Basel

0378-5866/13/0355-0373\$38.00/0

E-Mail karger@karger.com

www.karger.com/dne

\section{Introduction}

Spinal cord injury and/or regeneration-related gene NO 39 (SCIRR39) is an identified upregulated gene in rat spinal cord primary neuron injury and/or regeneration process through improved subtractive hybridization [unpubl. data]. Bioinformatics analysis revealed that SCIRR39 is Rattus norvegicus ribonuclease/angiogenin inhibitor 1 (AY240937.2) with only two amino acid residues difference from Homo sapiens ribonuclease/angiogenin inhibitor 1 (RNH1) that is known as a member of leucine-rich repeats motif (LRR) protein superfamily [1]. Leucinerich repeats are found in proteins with diverse functions. In the nervous system, members of the LRR superfamily, such as Erbin, Lrig3, Lrrn1, and AMIGO, have been isolated and found to be involved in neural development [25]. SCIRR39 is almost entirely composed of 13 leucinerich repeats, which might be involved in some unknown biological functions like other structurally similar proteins besides inhibiting ribonuclease (RNase) and angiogenin activities. It is generally considered that the injury regeneration process is likely to be a partial repeat of the individual development process. The change of SCIRR39 expression after primary neuron injury might provide clues to understand the relationship between SCIRR39 and development, and hint a potential role in injury regeneration.

\section{C.F. Zhao and Y. Liu contributed equally to this work.}


Table 1. Primer pairs used in PCR experiments

\begin{tabular}{|c|c|c|c|c|}
\hline Gene & $\begin{array}{l}\text { GenBank } \\
\text { accession No. }\end{array}$ & Sense primer $\left(5^{\prime} \rightarrow 3^{\prime}\right)$ & Antisense primer $\left(5^{\prime} \rightarrow 3^{\prime}\right)$ & $\begin{array}{l}\text { Product } \\
\text { size, bp }\end{array}$ \\
\hline C3-transferase-full* & NC003030.1 & GACAGATCTATGATAGGACAAACAGCTG & TCGAAGCTTCTACCTCTTTAAATTCAA & 1,185 \\
\hline C3-transferase & NC003030 & GGAGAACCGTTGATAACATTGG & CATTTCAGCCTCTACTTGACG & 293 \\
\hline SCIRR39 & AY240937 & TGCTCTCTGTCTGCTGCTTC & CCGCAAGATTCCAGTTTCA & 176 \\
\hline RhoA & NM057132 & TATGGCAAACAGGATTGGC & TTCACAAGATGAGGCACCC & 146 \\
\hline ROCK II & NM013022 & CAGCAGAAATGGGTTAGTCG & GCTTGGTTTGTTTGGAGCA & 156 \\
\hline
\end{tabular}

* The primer pairs for full length of C3-transferase and SCIRR39 were used for plasmids construction. Other primer pairs were applied for real-time quantitative PCR.

The transition of neuronal precursors from a proliferating state to a completely differentiated phenotype represents a crucial event that characterizes the central nervous system (CNS) developmental program. Neuroblasts permanently lose the ability to enter the cell cycle, migrate to their specific positions and undergo major morphological changes, which result in the establishment of a complex neuronal network [6-9]. Studies have shown that small GTPases of the Ras homolog gene family (Rho) are important regulators of the actin cytoskeleton, which play a crucial role during every stage of the neuronal differentiation program [10-13]. Rho proteins are intracellular signal transducers that regulate the transition from an active GTP-bound state to an inactive GDP-bound form under tight regulation. Effector proteins change their activity upon the interaction with the GTP-bound form. Rho GTPases exert functions through a complex network of these effector proteins [14].

To investigate the potential role of SCIRR39 in the processes after CNS injury and developmental program, we identified that SCIRR39 was expressed in rat pheochromocytoma PC12 line, a commonly used model for neuronal differentiation. SCIRR39 positively regulated neurite extension in NGF-treated PC12 cells. We also identified that RhoA pathway was regulated by SCIRR39 in PC12 cells. These results suggest the important role of SCIRR39 in neural differentiation and may provide a new therapeutic target for treatment of injury in the CNS.

\section{Material and Methods}

\section{Plasmids}

The plasmid used for transfection was made by cloning a PCR fragment of rat SCIRR39 and C3-transferase CDNA sequence from pCMV-Tag2B-SCIRR39 or pcDNA3.1-C3 (a gift of Geron Co.) into the pEGFP-N1/pECFP-N1 (Clontech Laboratories, Inc., Palo Alto, Calif., USA) with primer pairs as described in table 1, to produce a fusion protein with the EGFP/ECFP domain. Amplification conditions: 35 cycles of $95^{\circ} \mathrm{C}$ for $30 \mathrm{~s}, 63^{\circ} \mathrm{C}$ for $30 \mathrm{~s}$, and $72^{\circ} \mathrm{C}$ for $90 \mathrm{~s}$ in a $50-\mu \mathrm{l}$ reaction system.

\section{Cell Culture and Transfection}

PC12 (a stock of American Type Culture Collection) was a gift from Dr. S.Q. Jing, Amgen Co., USA. PC12 cells were grown in DMEM supplemented with $10 \%$ horse serum and $5 \%$ fetal calf serum on poly-D-lysine-coated plates. Differentiation was induced for the indicated times with medium containing $50 \mathrm{ng} / \mathrm{ml}$ NGF (Sigma, St. Louis, Mo., USA). PC12 cells that have at least one neurite 2-fold longer than the main cell body axis were identified as neurite-bearing cells [15]. PC12 cells were transfected using Lipofectamine 2000 (Invitrogen, Grand Island, N.Y., USA) according to the manufacturer's specifications.

Construction of SCIRR39 shRNA Plasmid and Cell Infection

Rat SCIRR39 cDNA sequences were used as candidate small hairpin RNAs. LV-SCIRR39, for lentivirus expression of SCIRR39 shRNA, was constructed by annealing the following oligonucleotides: 5 '-TCAGCTGGTACTGTACGACATTCTCGAGAATGTCGTACAGTACCAGCTGTTTTTTC- $3^{\prime}$ and $5^{\prime}$-TCGAGAAAAAACAGCTGGTACTGTACGACATTCTCGAGAATGTCGTACAGTACCAGCTGA-3' and ligating to linearized pGCSILPUR vector after Age I and EcoR I digestion. Lentiviruses carrying GFP were generated as described (Shanghai Genechem Co., Ltd). PC12 cells were infected with lentivirus at a multiplicity of infection of 5 per cell. In control or SCIRR39 shRNA lentivirus infected PC12 cells, approximately $80 \%$ of cells were GFP-positive. To quantify the effects of shRNA on neurite extension, only GFP-positive PC12 cells were counted. In rescue experiments, we analyzed the percentage of neurite-bearing PC12 cells after transfection/infection with the SCIRR39-CFP and SCIRR39 shRNAGFP constructs. Only CFP and GFP double-positive PC12 cells were counted. To minimize the subjective assessments, 20 microscopic fields were randomly selected in each group and then digital photographs taken. The neurite-bearing cells in those photographs were separately numbered by another unwitting experimenter. 


\section{Antibodies}

The following antibodies were used: rabbit polyclonal antiRNH1 (Sigma); rabbit polyclonal anti-active caspase-3 (Sigma); rabbit polyclonal anti-RhoA (Abcam, Cambridge, Mass., USA); rabbit polyclonal anti-ROCK II (Abcam); rabbit monoclonal antiFyn (Bioworld Technology, Inc., St. Louis Park, Minn., USA), rabbit monoclonal anti-p-Fyn (Y420) (Sigma) and mouse monoclonal anti-GAPDH (Sigma).

\section{Cell Proliferation Assay}

PC12 cells were seeded in a 96-well plate and then transiently transfected or infected with various recombinant plasmids or lentivirus. 1, 2 and 3 days later, cell proliferation was determined by a commercial Cell Counting Kit (CCK-8; Dojindo Molecular Technologies, Gaithersburg, Md., USA), which is based on the detection of dehydrogenase activity in viable cells. $10 \mu \mathrm{l}$ of CCK- 8 reagent were added to each well and incubated at $37^{\circ} \mathrm{C}$ for $2 \mathrm{~h}$, until the media turned yellow. Absorbance was measured at $450 \mathrm{~nm}$ in an automated ELISA instrument.

\section{Cell Cycle and Apoptosis Assay}

Flow cytometric analysis of DNA content was used to assess the effect of SCIRR39 on cell cycle. Cells, at $72 \mathrm{~h}$ after transfection of pSCIRR39-GFP or infection of SCIRR39 shRNA lentivirus, were fixed in $70 \%$ ethanol at $-20^{\circ} \mathrm{C}$. After washing with PBS, cells were treated with RNase A and stained with propidium iodide. The DNA content was evaluated using a FACSCalibur flow cytometer (BD Biosciences, San Jose, Calif., USA). 20,000 cells were analyzed per sample.

Annexin V assay was used to detect apoptotic cells. NGF-treated cells were harvested at $48 \mathrm{~h}$ after transfection of pSCIRR39-GFP or $72 \mathrm{~h}$ after infection of SCIRR39 shRNA lentivirus, and washed with PBS. The cells were then stained using an Annexin V-TRITC Apoptosis Detection Kit (Invitrogen). Cells transfected with pEGFP-N1 or infected by GFP lentivirus served as control. The cells were analyzed immediately after staining using FACSCalibur flow cytometer and CellQuest Pro software. 20,000 cells were analyzed per sample.

\section{Immunofluorescence Microscopy}

Cells were fixed with $4 \%$ paraformaldehyde in PBS for $10 \mathrm{~min}$, and permeabilized with $0.1 \%$ Triton X-100 for 5 min. Immunofluorescence staining was performed using the above-described antibodies, followed by incubation with appropriate fluorescentconjugated secondary antibodies (Invitrogen). Homologous IgG was used as isotype controls. Polymeric F-actin was detected with phalloidin TRITC (Sigma), and nuclei were stained with Hoechst 33258 (Sigma).

Cells were imaged using a Zeiss LSM710 confocal microscope. Stacks were created from 7 to 12 slices at different focal levels $(0.36$ $\mu \mathrm{m}$ apart), and analyzed with ZEN software (Zen 2008 light edition; Carl Zeiss MicroImaging, Inc., Thornwood, N.Y., USA).

\section{Real-Time Quantitative PCR}

Total cellular RNAs were extracted using RNA extraction kit (Tiangen Co., Beijing, China) and $2 \mu \mathrm{g}$ of total RNA from each sample was used for reverse transcription. Real-time quantitative PCR was performed using SYBR Green Real-Time PCR Master Mix (Roche) with primer pairs described in table 1 in the ABI 7500 sequence detector system (Applied Biosystems, Foster City, Calif., USA). The relative expression of $R$. norvegicus Fyn, RhoA and

SCIRR39 Increased the Neurite Extension in PC12 by RhoA
ROCK II mRNAs was assessed by the comparative cycle threshold method and normalized to glyceraldehyde-3-phosphate dehydrogenase (GAPDH) mRNA (table 1).

\section{Immunoblotting}

For immunoblots an equal amount of proteins from total cell lysates was separated by reducing SDS-PAGE and transferred to nitrocellulose membrane (Osmonics, Inc., Minnetonka., Minn., USA), and then incubated with primary antibodies and horseradish peroxidase-labeled secondary antibodies. The blots were washed and visualized using an enhanced chemiluminescence immunoblotting detection kit (Vigorous, Beijing, China). The optical densities of the respective protein bands were quantified with an analyzer equipped with the Quantity One software package and normalized to a GAPDH internal control.

\section{Rho Activity Assay}

NGF-treated cells were lysed and the contents of RhoA-GTP and total RhoA were determined as the protocol of Rho activity assay kit (Enzo Life Sciences, Farmingdale, N.Y., USA) according to the manufacturer's instructions.

\section{Statistical Analysis}

Data were represented as mean \pm SEM from at least three independent experiments. Student's t test was used to evaluate the difference and $\mathrm{p}<0.05$ was considered statistically significant.

\section{Results}

\section{SCIRR39 Is Expressed in PC12 Cells and Increased in Differentiated Cells}

Real-time quantitative PCR and Western blotting assays were performed to detect the expression of SCIRR39 in PC1 2 cells. Results showed that SCIRR39 was expressed in normal PC12 cells, and was increased in the differentiated cells after NGF treatment (fig. 1a-c).

\section{SCIRR39 Inhibits the Proliferation of PC12 Cells}

To study the role of SCIRR39 in PC12 cells, PC12 cells were transfected with empty green fluorescent protein (GFP) vector or SCIRR39-GFP plasmid, and high expression of SCIRR39 mRNA and protein was confirmed in the SCIRR39-GFP transfected PC12 cells by real-time quantitative PCR and Western blotting (fig. 2a, b). As shown in figure $2 c$, the proliferation of SCIRR39-GFP transfected cells was slower than that of control cells.

To further confirm the role of SCIRR39 in PC12 cells, we developed SCIRR39 lentiviral shRNA. Compared with the corresponding control, SCIRR39 shRNA was able to significantly decrease SCIRR39 expression in PC12 cells (fig. 2a, d, e). As we expected, the proliferation of PC12 cells after SCIRR39 knockdown was slightly faster than that of control cells (fig. 2f). 
The effect of SCIRR39 on cell proliferation was further confirmed by cell cycle analysis using flow cytometry (FCM). In SCIRR39-GFP transfected cells, the cell proportion in the $S$ phase was reduced, and growth arrest in the G0/G1 phases was observed, indicating the inhibition of cell proliferation. However, SCIRR39 knockdown did not affect cell cycle progression of PC12 cells (fig. 2g, h). Moreover, SCIRR39-GFP overexpression had no significant role in apoptosis that was evaluated by the blotting of anti-activated caspase-3 (fig. 2i, j). Using the Annexin $\mathrm{V}$ assay, we found that SCIRR39 knockdown increased the portion of apoptotic cells from 1.91 to $4.03 \%$, whereas SCIRR39 overexpression did not have any significant effect on apoptosis (fig. 2k, 1).

\section{SCIRR39 Increases Neurite Extension in NGF-Treated PC12 Cells}

We treated different cell transfectants with NGF and examined the morphological changes by phalloidin
(PHD) staining. After 2 days of NGF treatment, 90\% of SCIRR39-GFP-positive PC12 cells was induced to differentiate into neuron-like phenotype and neurites of cells were 2 -fold longer than cell body, while most of vectorGFP-positive cells displayed round morphology, with rare and short neurites (fig. 3a, b). No morphological differentiation was detected in SCIRR39-GFP-positive PC12 cells without NGF treatment (data not shown). We also verified the role of SCIRR39 in neurite extension by knockdown experiment. Here we found SCIRR39 shRNA significantly inhibited neurite extension, since the percentage of neurite-bearing cells was obviously decreased after SCIRR39 inhibition (fig. 3c, d). Thus these observations indicated that SCIRR39 might act as an enhancer of neurite extension. These effects were not dependent on the particular construct, because similar results were also obtained with YFP and FLAG fusions, as well as another effective SCIRR39 shRNA. Considering the common offtarget effect of shRNA, rescue experiments were per-

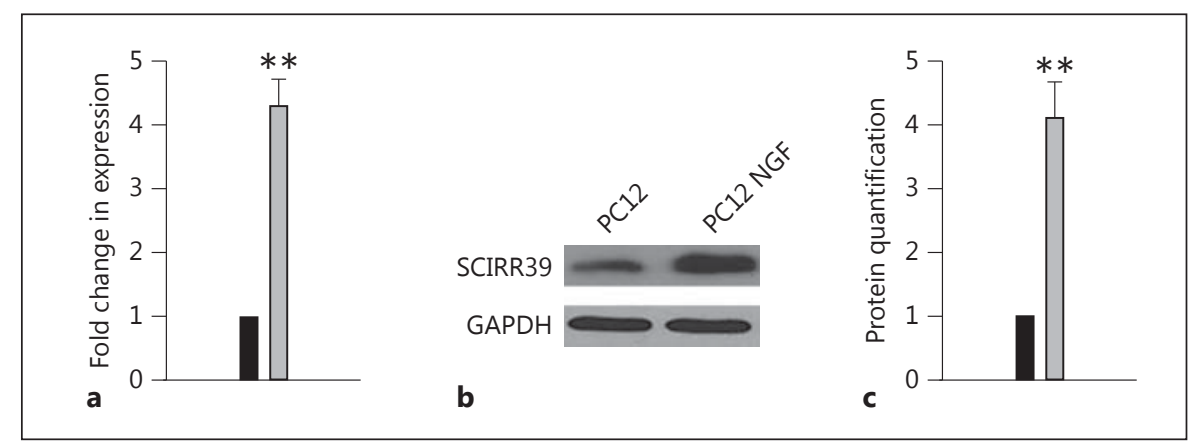

Fig. 1. Expression of SCIRR39 in PC12 cells. Differentiation was induced by $50 \mathrm{ng} / \mathrm{ml} \mathrm{NGF}$ for $72 \mathrm{~h}$. a Real-time quantitative PCR analysis of SCIRR 39 mRNA expression in undifferentiated and differentiated PC12 cells. GAPDH gene was used as an internal control. The bar graphs showed the average of three independent experiments, which were represented as mean \pm SEM. ${ }^{* *} \mathrm{p}<0.01$ (Student's t test). b Detection of SCIRR39 protein expression by
Western blotting. GAPDH was used as the internal control. c The quantification of SCIRR39 expression. SCIRR39 protein shown in $\mathbf{b}$ was quantified after a densitometric scan and normalized to GAPDH. The results were expressed as the mean \pm SEM from three independent experiments. ${ }^{* *} \mathrm{p}<0.01$ (Student's t test). Black: PC12; grey: PC12 (NGF).
Fig. 2. a-I Effects of SCIRR39 on cell proliferation, cell cycle and apoptosis. PC12 cells were transfected with SCIRR39-GFP and GFP plasmids or infected with lentiviruses of SCIRR39 shRNA and LVGFP control. a Real-time quantitative PCR analysis of SCIRR39 mRNA expression. GAPDH gene was used as an internal control. b, d, i Western blotting was used to detect overexpressed SCIRR39GFP (77 kDa), endogenous SCIRR39 (50 kDa) and active caspase-3 (in NGF-treated cells). GAPDH was used as the internal control. c, f Cell proliferation of corresponding PC12 cells was measured by a commercial Cell Counting Kit at 1,2 and 3 days after transfection or infection. After incubation, absorbance was measured at $450 \mathrm{~nm}$ in an automated ELISA instrument. e, $\mathbf{j}$ The quantification of protein expression. SCIRR39 and active caspase- 3 proteins shown in $\mathbf{d}$ and $\mathbf{i}$ were quantified after a densitometric scan and normalized to GAPDH. The results were expressed as the mean \pm SEM from three independent experiments. Black: LVGFP; grey: SCIRR39 shRNA in e. $\mathbf{g}, \mathbf{h}$ Cell cycle of corresponding PC12 cells was analyzed by propidium iodide staining using FCM. k, I Using TRITC-Annexin V assay, the apoptotic cells in NGF-treated PC12 cells were detected at $48 \mathrm{~h}$ after transfection or $72 \mathrm{~h}$ after infection. The percentage of Annexin V-TRITC-positive cells to total cells was shown in the bar graphs. The data were calculated based on at least three independent experiments and represented as mean \pm SEM. ${ }^{* *} \mathrm{p}<0.01$ (Student's t test). 


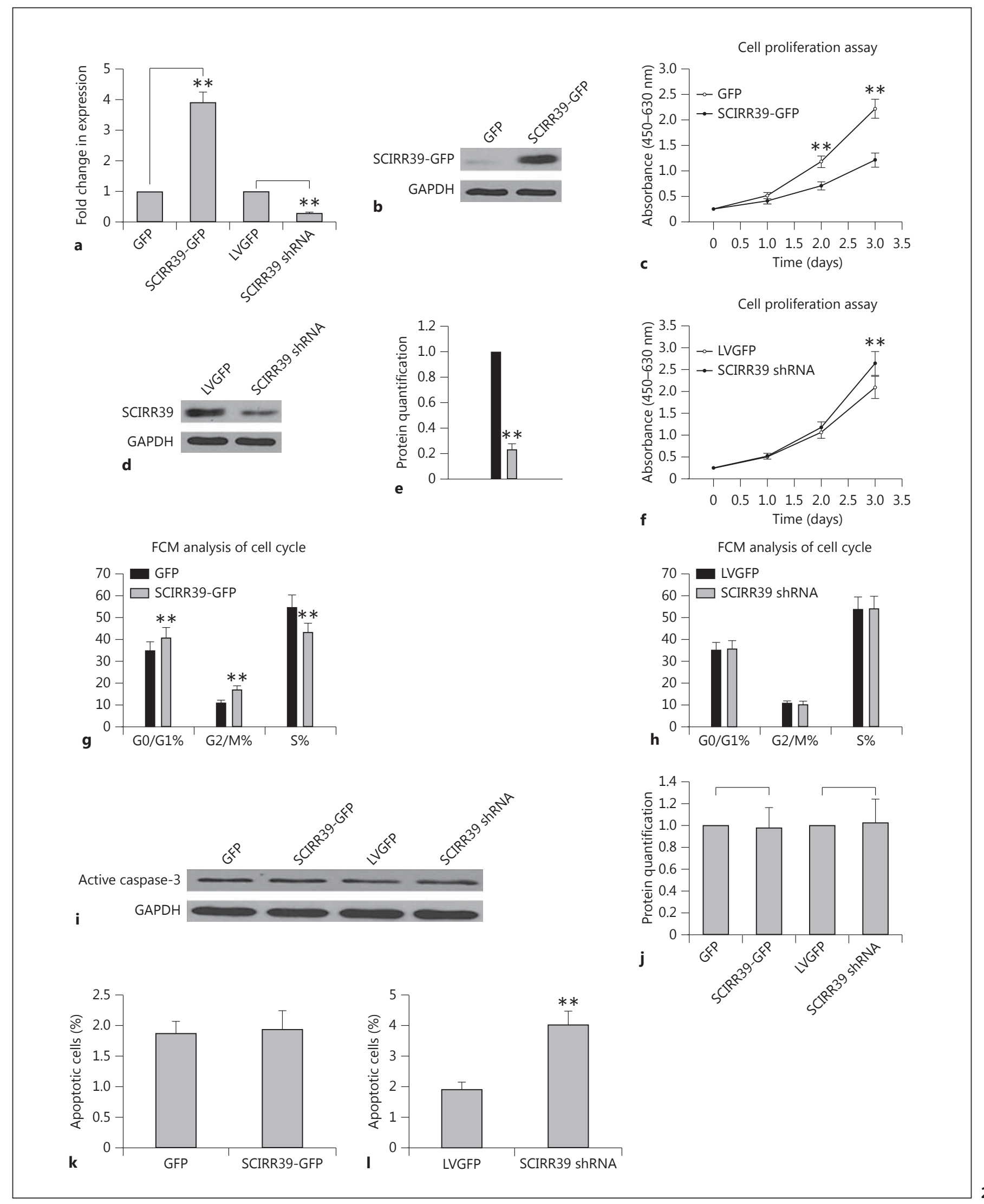




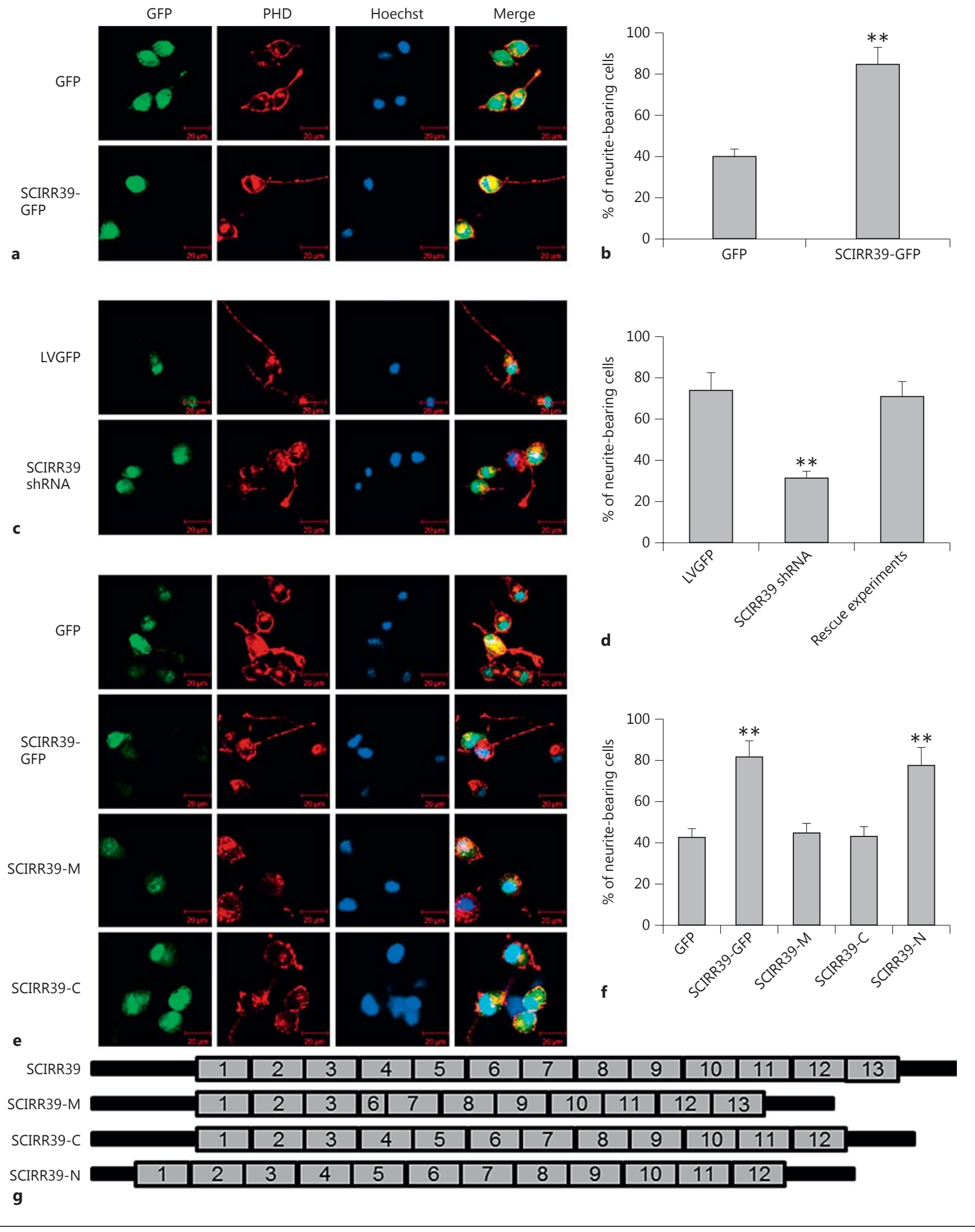


formed in SCIRR39 knockdown PC12 cells. The reduced percentage of neurite-bearing cells by SCIRR39 knockdown was restored to the control level after overexpression of SCIRR39-GFP in NGF-treated PC12 cells (fig. 3d).

To explore which regions of SCIRR39 are involved in enhancing neurite extension, we also tested the activity of some SCIRR39-GFP mutants. Two mutants, SCIRR39$\mathrm{M}$ (contains a truncation from 141AA to 211AA) and SCIRR39-C (contains a truncation from 397AA to 424AA), failed to promote neurite extension as the fulllength protein, which showed similar results as control vector cells (fig. $3 \mathrm{e}-\mathrm{g}$ ), thus suggesting that both the middle part and C-terminal of SCIRR39 are necessary for full activity. However, the $\mathrm{N}$-terminal (from 1AA to 34AA) was not required for the similar results from the cells with overexpression of full-length protein (fig. $3 \mathrm{f}$ ).

\section{SCIRR39 Regulates RhoA and Fyn}

A strong candidate signaling pathway involving neurite extension in PC12 cells is RhoA-ROCK. RhoA is a crucial mediator for neurite extension after NGF treatment [16]. In particular, reduced RhoA-GTP level is required for neurite extension in PC12 cells $[17,18]$. Moreover, among the different effectors of RhoA, Rho kinases (ROCK) are believed to play a particularly important role in transducing neurite-inhibitory signals [19-21]. Therefore, to gain further mechanistic insight into the role of SCIRR39 in PC12 cells, we examined whether SCIRR39 affects the RhoA-ROCK pathway. Here we found that SCIRR39 overexpression remarkably decreased the level of active RhoA in NGF-treated PC12 cells, while RhoAGTP level was upregulated after SCIRR39 knockdown

Fig. 3. a-g Effect of SCIRR39 on neurite extension. a, c, e PC12 cells were treated with NGF after transfection with GFP control, SCIRR39-GFP or two SCIRR39-GFP mutants, allowed to differentiate for $48 \mathrm{~h}$. In addition, PC12 cells were treated with NGF after infection with SCIRR39 shRNA or LVGFP lentivirus, allowed to differentiate for $96 \mathrm{~h}$. All cells were fixed and stained with PHD and analyzed by laser confocal microscope. Green: GFP; red: stained with PHD; blue: Hoechst 33258 staining. Bar: $20 \mu \mathrm{m}$. b, d, f The percentage of neurite-bearing PC12 cells that have at least one neurite 2 -fold longer than the main cell body axis was quantitatively analyzed. For each sample, 20 random fields were counted. The bar graphs showed the average of three independent experiments, which were represented as mean \pm SEM. All the samples are compared with PC12 cells that infected/transfected with LVGFP/pEGFP-N1. ${ }^{* *} \mathrm{p}<0.01$ (Student's t test). g Leucine-rich repeats motif domain structure (grey) of SCIRR39 protein and the truncated mutants.

SCIRR39 Increased the Neurite Extension in PC12 by RhoA (fig. 4a, b). No changes of RhoA mRNA and total protein were observed in all cell transfectants (fig. $4 \mathrm{a}, \mathrm{b}, \mathrm{g}, \mathrm{h}$ ).

The activity of RhoA is regulated by Fyn kinase [22, 23]. Increased Fyn expression and phosphorylation accelerate neurite extension in PC12 cell [23]. To test if SCIRR39 affects Fyn function, Fyn expression and phosphorylation were measured by Western blotting. SCIRR39 overexpression upregulated Fyn protein expression and its phosphorylation in NGF-treated PC12 cells (fig. 4c, d). Conversely, Fyn expression and its phosphorylation level were significantly reduced by SCIRR39 shRNA (fig. 4e, f). The similar change of Fyn mRNA after SCIRR39 treatments was confirmed using real-time quantitative PCR (fig. 4g, h).

In addition, we also examined the expression of ROCK II, an isoform of ROCK specifically expressed in PC12 cells, which is regulated by SCIRR39. ROCK II mRNA was downregulated (0.65-fold) in SCIRR39-GFP-positive cells, but slightly upregulated in SCIRR39 knockdown cells (fig. 4g, h), whereas no change of ROCK II protein was detected by Western blotting (fig. 4c-f).

\section{Neurite Extension-Inhibiting Activity of SCIRR39 shRNA Requires RhoA}

To confirm whether the SCIRR39 function in neurite extension involved RhoA, we forcedly expressed RhoA inhibitor C3-transferase (C3) [24] in SCIRR39 knockdown PC12 cells, and then treated with NGF. Although SCIRR39 shRNA dramatically inhibited neurite extension of PC12 cells, the reduced percentage of neuritebearing cells by SCIRR39 knockdown was restored to the control level after transfection of C3-transferase (fig. 5a, b). Moreover, many of SCIRR39 shRNA/C3-expressing cells showed long neurites compared with SCIRR39 knockdown alone cells (fig. 5a). The effects of C3-transferase overexpression and SCIRR39 shRNA were confirmed by real-time quantitative PCR (fig. 5 c). In addition, the RhoA pull-down assay showed that C3-transferase significantly neutralized the enhanced activity of RhoA-GTP induced by SCIRR39 knockdown (fig. 5d, e).

\section{Discussion}

The function of SCIRR39 has not been addressed, although its mRNA is enriched in the developing CNS and injured primary neuron (data not shown).

We found that SCIRR39 played crucial roles during the transition between proliferation and neurite extension in PC12 cells. SCIRR39 was expressed in PC12 cells 
Fig. 4. a-h Regulation of SCIRR39 to RhoA and Fyn. PC12 cells treated with SCIRR39GFP or SCIRR39 shRNA lentivirus were induced by NGF and allowed to differentiate. a RhoA GTP level in corresponding PC12 cells was detected by Western blotting. GFP and LVGFP were used as controls. $\mathbf{b}, \mathbf{d}, \mathbf{f}$ The quantification of protein expression. RhoA GTP, total RhoA, Fyn, phosphorylated Fyn (pFyn) and ROCK II proteins shown in $\mathbf{a}, \mathbf{c}$ and $\mathbf{e}$ were quantified after a densitometric scan and normalized to GAPDH. The results were expressed as the mean \pm SEM from three independent experiments. ${ }^{* *} \mathrm{p}<0.01$ (Student's t test). c, e Fyn, phosphorylated Fyn (pFyn) and ROCK II expression in NGF-treated PC12 cells transfected/infected with SCIRR39GFP/SCIRR39 shRNA lentivirus or control, were detected by Western blotting. GAPDH was used as the internal control. $\mathbf{g}, \mathbf{h}$ Real-time quantitative PCR analysis of Fyn, RhoA and ROCK II mRNA expression. GAPDH gene was used as the internal control. The bar graphs showed the average of three independent experiments, which were represented as mean \pm SEM. ${ }^{*} \mathrm{p}<0.05$ (Student's t test); ${ }^{* *} \mathrm{p}<0.01$ (Student's t test).

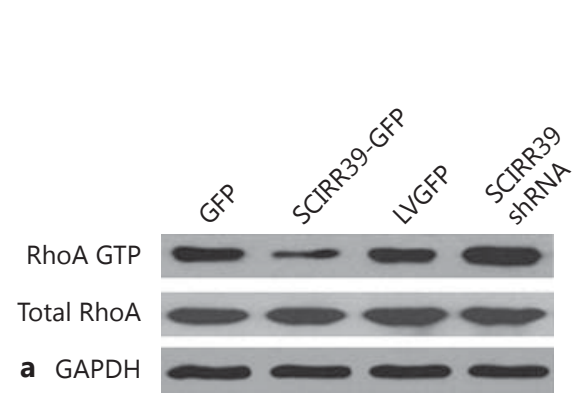

a GAPDH
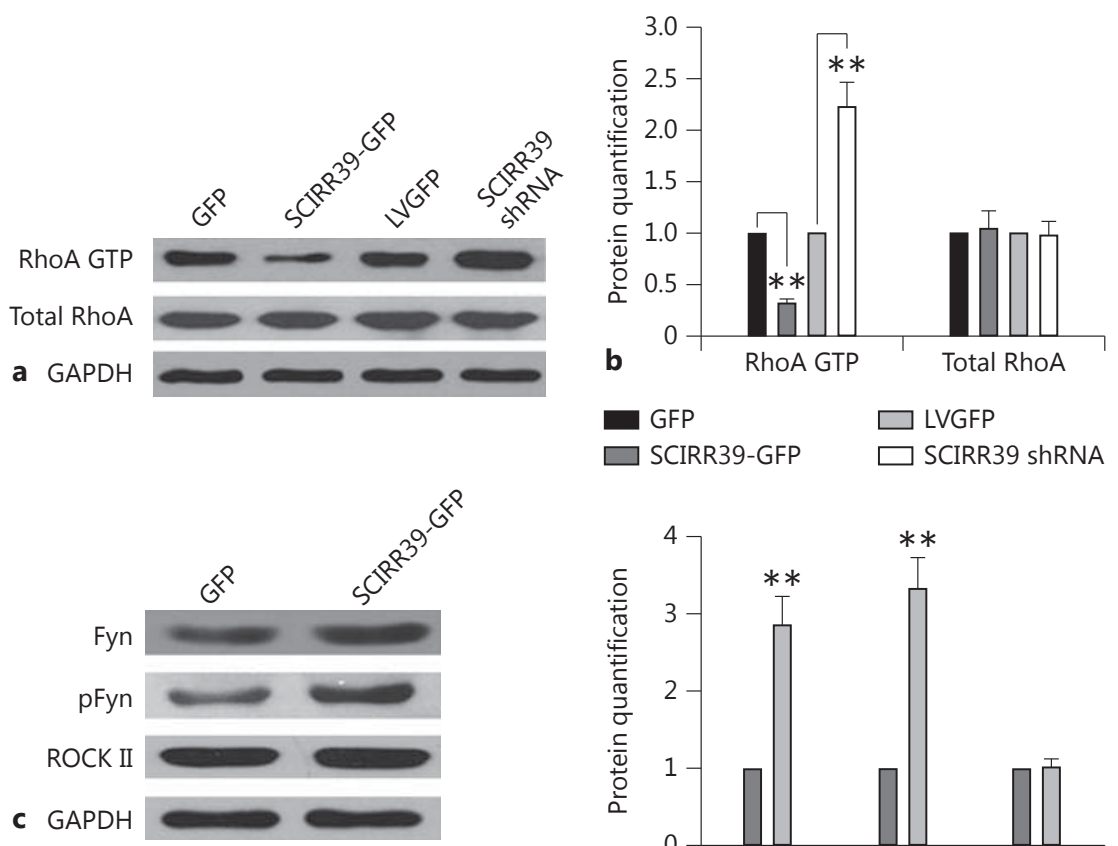

c GAPDH
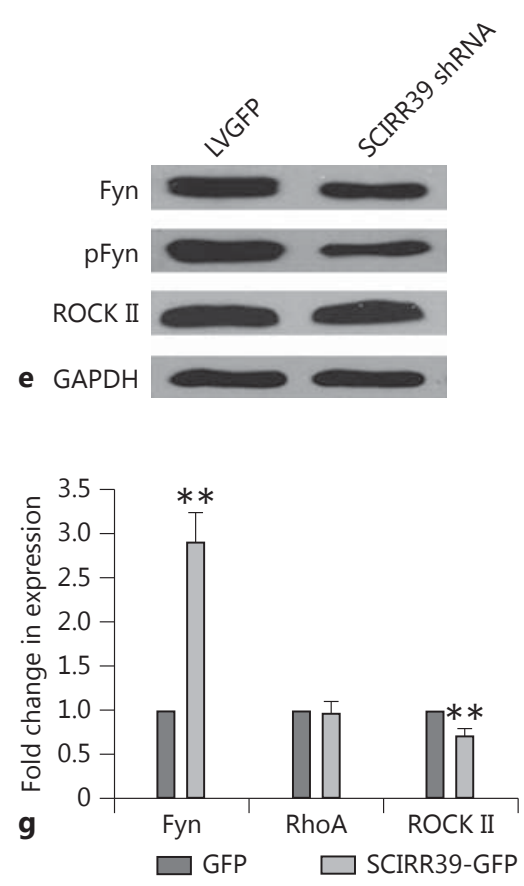
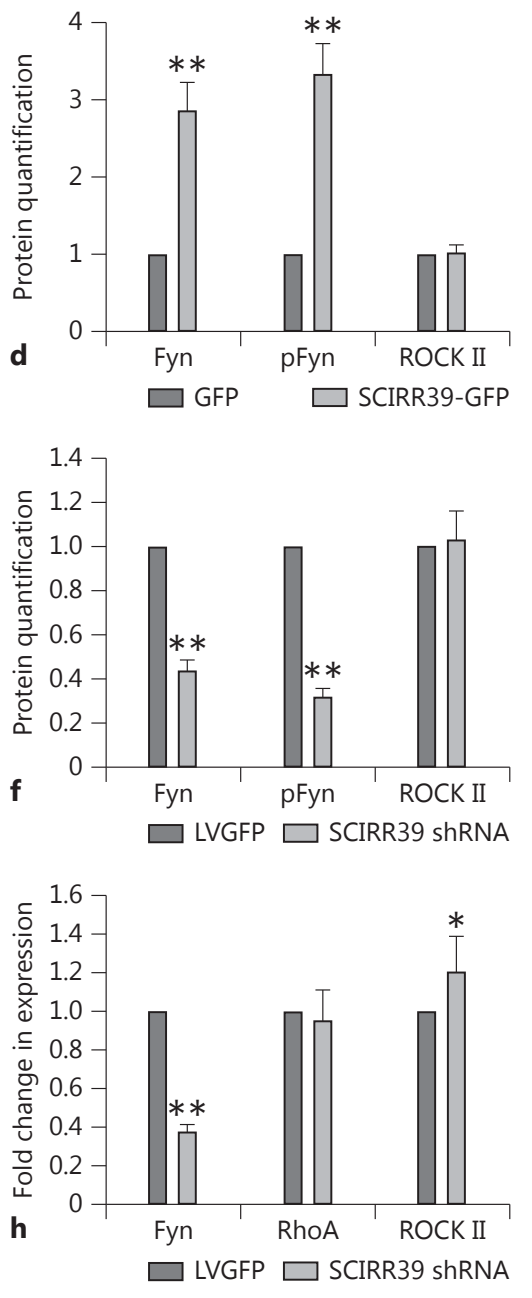

and was a positive regulator of neurite extension, an indicator of differentiation, after stimulation with the neurotrophin NGF. Loss of SCIRR39 using SCIRR39 shRNA led to decreased neurites length, whereas overexpression of SCIRR39 led to enhancement of PC12 neurite extension induced by NGF. Rescue experiments provided conclusive evidence that knockdown of SCIRR39 is responsible for the inhibition of neurite extension in NGF-treated PC12 cells. Those results suggest that the function of SCIRR39 may be related to the reg- 
Fig. 5. RhoA inhibition neutralizes the phenotype induced by SCIRR39 shRNA in NGF-treated PC12 cells. PC12 cells were infected with LVGFP or SCIRR39 shRNA lentivirus, in combination with C3-transferase-cyan fluorescent protein (CFP) or $\mathrm{CFP}$-empty vector. Cells were treated with NGF and allowed to differentiate for $96 \mathrm{~h}$. a Corresponding PC12 cells were immunofluorescence-stained. Green: GFP; red: stained with PHD; cyan: CFP; blue: Hoechst 33258 staining. Bar: $20 \mu \mathrm{m}$. b Quantitative analysis of neurite-bearing cells. For each sample, 20 random fields were counted. c Real-time quantitative PCR analysis of SCIRR39, C3-transferase mRNA expression in corresponding PC12 cells. GAPDH gene was used as the internal control. The bar graphs showed the average of three independent experiments, which were represented as mean \pm SEM. ${ }^{* *} \mathrm{p}<0.01$ (Student's t test). d RhoA GTP level in differential PC12 cells treated with LVGFP or SCIRR39 shRNA lentivirus, in combination with C3-transferase-CFP or CFPempty vector, were detected by Western blotting. LVGFP + CFP was used as control. e Quantification of protein expression. RhoA GTP and total RhoA protein shown in $\mathbf{d}$ was quantified after a densitometric scan and normalized to GAPDH. The results were expressed as the mean \pm SEM from three independent experiments. ** $\mathrm{p}<0.01$ (Student's t test).

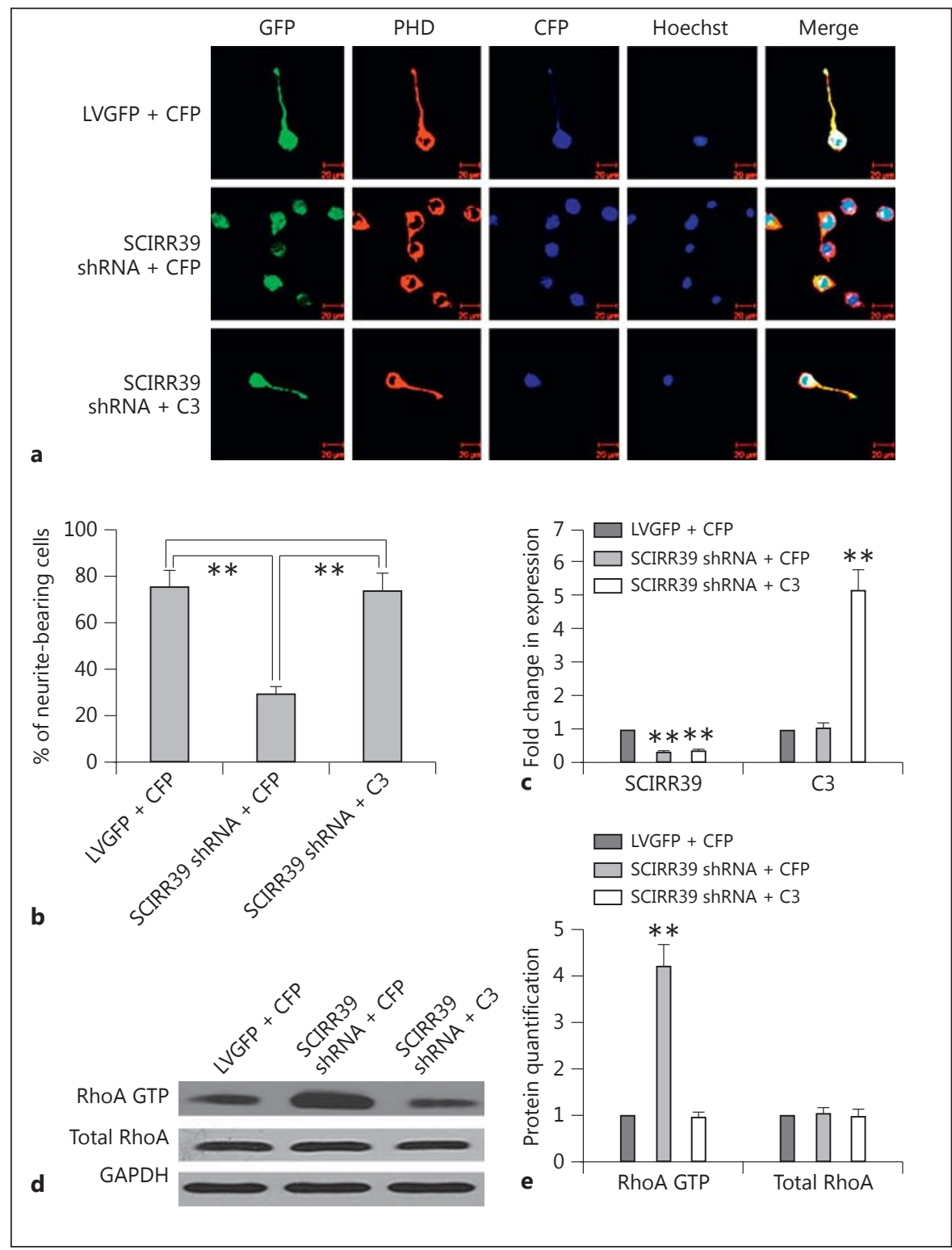

ulation of differentiation in NGF-induced PC12 cell. After exploring the relationship between structure and function of SCIRR39, we further identified which parts (141AA to 211AA and 397AA to 424AA) of SCIRR39 are crucial to promote neurite extension. Moreover, the possibility that any shortening of SCIRR39 blocks its effects is eliminated, for the $\mathrm{N}$-terminal sequence of it is dispensable.

The growth arrest in the G0/G1 phases was observed in the SCIRR39 overexpressing cells, which supports the result of cell proliferation assay in PC12 cells. We consider that SCIRR39 enhances differentiation in part by reducing time in cell division in view of the decrease of $S$ phase in SCIRR39 overexpression cells. However, cell cycle was not affected by SCIRR39 knockdown in PC12 cells. It has been known that RNase A can be endowed with cytotoxic activity by evading RNH1 $[25,26]$. Therefore, we speculate that the cytotoxicity of RNase A caused by SCIRR39 knockdown may influence cell cycle distribution and then offset the effect of SCIRR39 shRNA. By the same token, the increased portion of apoptotic cells in SCIRR39 knockdown cells (Annexin V assay) might be due to the decrease of SCIRR39, but not the involvement of caspase- 3 activation. 
To understand the molecular mechanism of SCIRR39, we investigated the downstream signaling molecules that are known to be involved in differentiation, like Fyn and RhoA. Fyn is a member of the Src family of tyrosine kinases and is involved in both brain development and adult brain function [27]. Recent studies have identified that the tumor suppressor PTEN, an interacting protein of RNH1 [28], controls Fyn phosphorylation [29], while the status of Fyn could downregulate RhoA activity and result in PC12 cell differentiation [23, 30, 31]. Fyn disruption implicates in the inhibition of PC12 cell differentiation, whereas activation of Fyn by cross-linking of F3/ contactin or integrin $\alpha_{6} \beta_{1}$ promotes cell differentiation [32-34]. In the present study, the expression and phosphorylation status of Fyn were upregulated in the SCIRR39 overexpressing PC12 cells, while the level of activated RhoA-GTP was reduced. Thus we speculate that SCIRR39 might regulate the activity of Fyn through the interaction with PTEN, and then inhibit the activity of RhoA. The commonly accepted view is that PC12 cell differentiation depends on the inhibition of RhoA [12, 35, 36]. Therefore, the decreased RhoA activity caused by SCIRR39 increases differentiation in PC12 cells. Previous studies revealed that axon growth and regeneration are blocked by RhoA signaling after CNS injury $[37,38]$, thus we predict the potential neuroprotective role of SCIRR39 in neuron injury.

Importantly, we also found that the inhibitory effect of SCIRR39 shRNA on neurite extension was substantially reversed by the overexpression of RhoA inhibitor C3tranferase, indicating that RhoA acts as important regulator for neurite extension in the downstream of SCIRR39.

Among the downstream effectors of RhoA, ROCK is known to play a crucial role in inhibiting differentiation of PC12 cells. When treated with NGF, it was shown that the inactivation of RhoA is accompanied by the dissociation from ROCK [16]. In this study, although ROCK II mRNA was downregulated by SCIRR39 overexpression but upregulated by SCIRR39 shRNA, the ROCK II protein seemed not to be affected by SCIRR39 overexpres- sion or knockdown while the reason was unclear. The results suggest that SCIRR39 function in neuronal differentiation may be due to RhoA but independently of ROCK II, because it has been reported that TTC 3 might act in neuronal differentiation via the regulation of RhoA activity but independently of ROCK [15]. Thus, we speculate that the neurite extension induced by NGF can be regulated by RhoA activation involving ROCK or not.

In addition to inducing morphologic differentiation via suppression of RhoA activity, SCIRR39 could also stimulate neurite extension by acting as an antioxidant. The antioxidant activity of RNH1 contributed by 32 cysteine residues is able to protect cells from peroxidative injuries [39]. In addition, antioxidant promotes PC12 cell differentiation via triggering comprehensive antiapoptotic and molecular chaperone responses [40, 41]. Consistent with these data, SCIRR39 would promote differentiation of PC12 cells after NGF treatment not only because of the decrease of activated RhoA-GTP, but also by the antioxidant activity. Moreover, the protective action of antioxidant in neuron is important in the secondary injury $[42,43]$, thus upregulation of SCIRR39 in primary neuron injury and/or regeneration process may act to minimize the secondary neurological damage that occurs during the acute phase after CNS injury.

In summary, we have provided in vitro evidence that SCIRR39 is a positive regulator for neurite extension of PC12 cells after NGF treatment, whose effect is involved in RhoA signaling. Thus, SCIRR39 may contribute to the regeneration of CNS injury by specifically altering the neuronal differentiation program.

\section{Acknowledgments}

This work was supported by grants from the Chinese National Basic Research Program (grant No. 2009CB918301), National Natural Science Foundation of China (grant No. 81301067), China Postdoctoral Science Foundation (grant No. 2013M532218), and Beijing Municipal Natural Science Foundation (grant No. 5112027).

\section{References}

1 Nadano D, Yasuda T, Takeshita H, Uchide K, Kishi K: Purification and characterization of human brain ribonuclease inhibitor. Arch Biochem Biophys 1994;312:421-428.

-2 Tao Y, Dai P, Liu Y, Marchetto S, Xiong WC, Borg JP, Mei L: Erbin regulates NRG1 signaling and myelination. Proc Natl Acad Sci USA 2009;106:9477-9482.
3 Zhao H, Tanegashima K, Ro H, Dawid IB: Lrig3 regulates neural crest formation in $\mathrm{Xe}$ nopus by modulating Fgf and Wnt signaling pathways. Development 2008;135:1283-1293.

4 Andreae LC, Peukert D, Lumsden A, Gilthorpe JD: Analysis of Lrrn1 expression and its relationship to neuromeric boundaries during chick neural development. Neural Dev 2007;2:22.
5 Kuja-Panula J, Kiiltomaki M, Yamashiro T, Rouhiainen A, Rauvala H: AMIGO, a transmembrane protein implicated in axon tract development, defines a novel protein family with leucine-rich repeats. J Cell Biol 2003;160: 963-973. 
6 Kawauchi T, Hoshino M: Molecular path- 19 Hirose M, Ishizaki T, Watanabe N, Uehata M, ways regulating cytoskeletal organization and morphological changes in migrating neurons. Dev Neurosci 2008;30:36-46.

7 Goffinet AM: Events governing organization of postmigratory neurons: studies on brain development in normal and reeler mice. Brain Res 1984;319:261-296.

8 Colomb S, Joly W, Bonneaud N, Maschat F: A concerted action of Engrailed and Gooseberry-Neuro in neuroblast 6-4 is triggering the formation of embryonic posterior commissure bundles. PLoS One 2008;3:e2197.

9 Kim Y, Comte I, Szabo G, Hockberger P, Szele FG: Adult mouse subventricular zone stem and progenitor cells are sessile and epidermal growth factor receptor negatively regulates neuroblast migration. PLoS One 2009; 4:e8122.

10 Iwai T, Saitoh A, Yamada M, Takahashi K, Hashimoto E, Ukai W, Saito T: Rhotekin modulates differentiation of cultured neural stem cells to neurons. J Neurosci Res 2012;90: 1359-1366.

11 Da Silva JS, Dotti CG: Breaking the neuronal sphere: regulation of the actin cytoskeleton in neuritogenesis. Nat Rev Neurosci 2002;3: 694-704.

$\checkmark 12$ Govek EE, Newey SE, Van Aelst L: The role of the Rho GTPases in neuronal development. Genes Dev 2005;19:1-49.

13 Kobayashi K, Masuda T, Takahashi M, Miyazaki J, Nakagawa $M$, Uchigashima $M$, Watanabe M, Yaginuma H, Osumi N, Kaibuchi K: Rho/Rho-kinase signaling pathway controls axon patterning of a specified subset of cranial motor neurons. Eur J Neurosci 2011;33:612-621.

14 Jaiswal M, Dubey BN, Koessmeier KT, Gremer L, Ahmadian MR: Biochemical assays to characterize Rho GTPases. Methods Mol Biol 2012;827:37-58.

15 Berto G, Camera P, Fusco C, Imarisio S, Ambrogio C, Chiarle R, Silengo L, Di Cunto F: The Down syndrome critical region protein TTC3 inhibits neuronal differentiation via RhoA and Citron kinase. J Cell Sci 2007;120: 1859-1867.

16 Nusser N, Gosmanova E, Zheng Y, Tigyi G: Nerve growth factor signals through TrkA, phosphatidylinositol 3-kinase, and Racl to inactivate RhoA during the initiation of neuronal differentiation of PC12 cells. J Biol Chem 2002;277:35840-35846.

17 Chakrabarti K, Lin R, Schiller NI, Wang Y, Koubi D, Fan YX, Rudkin BB, Johnson GR, Schiller MR: Critical role for Kalirin in nerve growth factor signaling through TrkA. Mol Cell Biol 2005;25:5106-5118.

18 Fernandez-Hernando C, Suarez Y, Lasuncion MA: Lovastatin-induced PC-12 cell differentiation is associated with RhoA/RhoA kinase pathway inactivation. Mol Cell Neurosci 2005;29:591-602.
Kranenburg O, Moolenaar WH, Matsumura F, Maekawa M, Bito H, Narumiya S: Molecular dissection of the Rho-associated protein kinase (p160ROCK)-regulated neurite remodeling in neuroblastoma N1E-115 cells. J Cell Biol 1998;141:1625-1636.

20 Da Silva JS, Medina M, Zuliani C, Di Nardo A, Witke W, Dotti CG: RhoA/ROCK regulation of neuritogenesis via profilin IIa-mediated control of actin stability. J Cell Biol 2003; 162:1267-1279.

21 Pacary E, Tixier E, Coulet F, Roussel S, Petit E, Bernaudin M: Crosstalk between HIF-1 and ROCK pathways in neuronal differentiation of mesenchymal stem cells, neurospheres and in PC12 neurite outgrowth. Mol Cell Neurosci 2007;35:409-423.

22 Liang X, Draghi NA, Resh MD: Signaling from integrins to Fyn to Rho family GTPases regulates morphologic differentiation of oligodendrocytes. J Neurosci 2004;24:71407149 .

23 Liu H, Nakazawa T, Tezuka T, Yamamoto T: Physical and functional interaction of Fyn tyrosine kinase with a brain-enriched Rho GTPase-activating protein TCGAP. J Biol Chem 2006;281:23611-23619.

24 Reshkin SJ, Murer H: Involvement of C3 exotoxin-sensitive $\mathrm{G}$ proteins (rho/rac) in PTH signal transduction in OK cells. Am J Physiol 1992;262:F572-F577.

25 Dickson KA, Dahlberg CL, Raines RT: Compensating effects on the cytotoxicity of ribonuclease A variants. Arch Biochem Biophys 2003;415:172-177.

-26 Futami J, Nukui E, Maeda T, Kosaka M, Tada $\mathrm{H}$, Seno M, Yamada H: Optimum modification for the highest cytotoxicity of cationized ribonuclease. J Biochem 2002;132:223-228.

27 Maness PF: Nonreceptor protein tyrosine kinases associated with neuronal development. Dev Neurosci 1992;14:257-270.

28 Kim YJ, Park SJ, Choi EY, Kim S, Kwak HJ, Yoo BC, Yoo H, Lee SH, Kim D, Park JB, Kim JH: PTEN modulates miR-21 processing via RNA-regulatory protein RNH1. PLoS One 2011;6:e28308

29 Dey N, Crosswell HE, De P, Parsons R, Peng Q, Su JD, Durden DL: The protein phosphatase activity of PTEN regulates SRC family kinases and controls glioma migration. Cancer Res 2008;68:1862-1871.

- 30 Jeon CY, Moon MY, Kim JH, Kim HJ, Kim JG, Li Y, Jin JK, Kim PH, Kim HC, Meier KE, Kim YS, Park JB: Control of neurite outgrowth by RhoA inactivation. J Neurochem 2012;120:684-698.

-31 Sebok A, Nusser N, Debreceni B, Guo Z, Santos MF, Szeberenyi J, Tigyi G: Different roles for RhoA during neurite initiation, elongation, and regeneration in PC12 cells. J Neurochem 1999;73:949-960.
32 Yamazaki Y, Horibata Y, Nagatsuka Y, Hirabayashi Y, Hashikawa T: Fucoganglioside a-fucosyl(a-galactosyl)-GM1: a novel member of lipid membrane microdomain components involved in PC12 cell neuritogenesis. Biochem J 2007;407:31-40.

-33 Pantera B, Bini C, Cirri P, Paoli P, Camici G, Manao G, Caselli A: PrPc activation induces neurite outgrowth and differentiation in PC12 cells: role for caveolin-1 in the signal transduction pathway. J Neurochem 2009; 110:194-207.

34 Nabemoto M, Mashimo M, Someya A, Nakamura H, Hirabayashi T, Fujino H, Kaneko M, Okuma Y, Saito T, Yamaguchi N, Murayama T: Release of arachidonic acid by 2 -arachidonoyl glycerol and HU210 in PC12 cells; roles of Src, phospholipase $\mathrm{C}$ and cytosolic phospholipase $\mathrm{A}_{2 \alpha}$. Eur J Pharmacol 2008; 590:1-11.

35 Obara Y, Ueno S, Yanagihata Y, Nakahata N: Lysophosphatidylinositol causes neurite retraction via GPR55, G13 and RhoA in PC12 cells. PLoS One 2011;6:e24284.

36 Katoh H, Aoki J, Ichikawa A, Negishi M: p160 RhoA-binding kinase ROKa induces neurite retraction. J Biol Chem 1998;273:2489-2492.

37 Yang P, Wen HZ, Zhang JH: Expression of a dominant-negative Rho-kinase promotes neurite outgrowth in a microenvironment mimicking injured central nervous system. Acta Pharmacol Sin 2010;31:531-539.

38 Conrad S, Schluesener HJ, Trautmann K, Joannin N, Meyermann R, Schwab JM: Prolonged lesional expression of RhoA and RhoB following spinal cord injury. J Comp Neurol 2005;487:166-175.

39 Cui XY, Fu PF, Pan DN, Zhao Y, Zhao J, Zhao BC: The antioxidant effects of ribonuclease inhibitor. Free Radic Res 2003;37: 1079-1085.

-40 Cassano S, Agnese S, D’Amato V, Papale M, Garbi C, Castagnola P, Ruocco MR, Castellano I, De Vendittis E, Santillo M, Amente S, Porcellini A, Avvedimento EV: Reactive oxygen species, Ki-Ras, and mitochondrial superoxide dismutase cooperate in nerve growth factor-induced differentiation of PC12 cells. J Biol Chem 2010;285:24141-24153.

41 Uittenbogaard M, Baxter KK, Chiaramello A: The neurogenic basic helix-loop-helix transcription factor NeuroD6 confers tolerance to oxidative stress by triggering an antioxidant response and sustaining the mitochondrial biomass. ASN Neuro 2012;2:e00034.

42 Ozgiray E, Serarslan Y, Ozturk OH, Altas M, Aras M, Sogut S, Yurtseven T, Oran I, Zileli M: Protective effects of edaravone on experimental spinal cord injury in rats. Pediatr Neurosurg 2012;47:254-260.

43 Khalatbary AR, Ahmadvand H: Neuroprotective effect of oleuropein following spinal cord injury in rats. Neurol Res 2012;34:44-51. 